\title{
Prácticas de lectura y escritura mediadas por las TIC en contextos educativos rurales*
}

\author{
Sabrina Bautista Villalobos,' Myriam Méndez de Cuéllar² \\ Fundación FES. Universidad de La Sabana (Colombia)
}

Recibido: diciembre 15 de 2014 - Revisado: enero 8 de 2015 - Aceptado: abril 30 de 2015

Referencia formato APA: Bautista, S., \& Méndez de Cuellar, M. (2015). Prácticas de lectura y escritura mediadas por las TIC en contextos educativos rurales. Revista Cientifica Guillermo de Ockham, 13(1), 97-107.

\section{Resumen}

El presente artículo de investigación presenta los resultados obtenidos en la investigación llevada a cabo en el año 2013 sobre las prácticas de lectura y escritura mediadas por las TIC, en el marco del programa Computadores para Educar. Este proyecto de investigación, indagó por los efectos que podría conllevar la implementación de herramientas tecnológicas y comunicativas en las prácticas de lectura y escritura de los estudiantes de contextos rurales, que no han tenido acercamiento con estas herramientas. Se utilizó una metodología cualitativa y se usó como técnica de recolección de información la observación no participante de actividades pedagógicas mediadas por las TIC. La observación se efectuó en el aula de clase, con estudiantes de veintiuna escuelas rurales y de básica primaria en el departamento de Cundinamarca. De lo anterior, se obtuvo un acercamiento a las transformaciones que se generan en las prácticas de lectura y escritura cuando se pasa del soporte físico al soporte digital, lo cual llevó a conclusiones acerca de las condiciones necesarias para que la implementación de este tipo de herramientas se ejecute de manera acertada y pertinente, en busca de un mejoramiento de la calidad educativa en contextos diferenciales, ser la escuela rural.

Palabras clave: Lectura, escritura, TIC, educación rural

\section{Reading and writing practices through ICT in rural education context}

\section{Abstract}

This research paper presents the results obtained in the research made in 2013 about reading and writing practices through ICT for the program "Computadores para Educar". This research project inquired about the effects that may be caused by the implementation of technological and communicative tools in reading and writing practices in remote areas where these had not been used by them. A qualitative methodology was used with an information collecting technique in non-participant observation of pedagogic activities through ICT. The observation was realised in classrooms with students in 21 rural and elementary schools in Cundinamarca. From this research, an approach to the transformations produced in reading and writing practices was obtained when changing from physical to digital,

* Este artículo se deriva del proyecto de investigación Nuevas formas de lectura y escritura con las TIC, alternativas en el aprendizaje del lenguaje para la escuela rural en Cundinamarca, desarrollado por la Fundación FES y la Universidad de La Sabana, en el marco del programa Computadores para Educar, del Ministerio de Tecnologías de la Información y las Comunicaciones MINTIC. Enero-diciembre de 2013.

1. Socióloga. Magíster en educación y en investigación educativa. Actualmente es coordinadora pedagógica de la Fundación FES. E-mail: sabrina. bautista@fessocial.org.

2. Licenciada en química y biología. Especialista en gerencia educativa y magíster en educación. Actualmente es directora del área de educación de la Fundación FES. E-mail:Myriam.mendez@fessocial.org. 
generating conclusions about the necessary conditions and recommendations so that the implementation of this kind of tool is successful and relevant for the improvement of quality education in differential contexts such as rural schools.

Keywords: Reading, writing, ICT, rural education

\section{Prática de leitura e escrita mediada pelas TIC em contextos educativos rurais}

\section{Resumo}

Este pesquisa artigo apresenta os resultados obtida em pesquisa segurado em 2013 sobre as practicas de leitura e escrita mediadas por TIC no ámbito do Programa Computadores para Educar. Este projeto de pesquisa investigou os efeitos que pode ter a implementação de ferramentas tecnológicas e de comunicação nas práticas de leitura e escrita dos estudantes fazer contextos rurais que não tinham aproximação com essas ferramentas A metodologia utilizada foi qualitativa, utilizando como técnica de coleta de dados não-participante de atividades educativas mediadas pela observação TIC. Observando a sala de aula com alunos de 21 escolas primárias rurais e básico, no departamento de Cundinamarca realizóen. Do acima exposto, uma abordagem para as mudanças que são gerados nas práticas de leitura e escrita quando se muda de hardware para mídia digital foi obtida, gerando conclusôes sobre as condiçôes e recomendaçóes necessárias para a execução de tais ferramentas abrir caminho bem sucedido e pertinente que permite a melhoria da qualidade educacional em contextos diferenciados, tais como escolas rurais.

Palavras-chave: Leitura, escrita, TIC, educação rural

\section{Introducción}

Desde la aparición en los años setenta de las Tecnologías de la Información y la Comunicación (TIC) y el internet en los ańos noventa (Sigalés, Momino, Meneses \& Badia, 2008), una preocupación de los gobiernos es estar a la vanguardia del desarrollo tecnológico con miras a lograr un acceso equitativo a la información -considerada como un derecho-y la formación de competencias tecnológicas de todos los ciudadanos de una nación.

Es así como en Colombia, el Ministerio de Tecnologías de la Comunicación y la Información (Mintic), por medio del programa Computadores para Educar ha dotado a las instituciones educativas públicas del país de una infraestructura tecnológica adecuada y ha capacitado a los docentes a través del diplomado Formación y Acceso para la Apropiación Pedagógica de las TIC. En este contexto, se dio la preocupación por investigar las transformaciones que se están dando en las instituciones educativas rurales de Cundinamarca con la llegada de las TIC, en especial, en aquellas formas de lectura y escritura que se presentan cuando se cambia del soporte físico al soporte digital.

Se enfatiza en las prácticas de lectura y escritura, puesto que estos procesos trascienden la simple codificación y decodificación, toda vez que al leer y al escribir, el ser humano involucra su capacidad para comprender e interpretar su propia realidad y asume una posición frente a la información que recibe, lo que lo lleva a un ejercicio más comprensivo del mundo en el que se encuentra. En la actualidad, surgen nuevas maneras de leer y escribir-propias de la sociedad del conocimiento y la información- que plantean otras formas de producir sentidos y vincularse a comunidades virtuales de conversación.

Este contexto plantea inesperados retos a la escuela, dado que la introducción de las TIC en los ambientes educativos transforma las formas de leer y escribir, los ambientes de aprendizaje y los tipos de textos que se leen, al tiempo que se producen $-y$, sobre todo, se complejizan y diversifican- nuevos modos de leer. Hoy en día y como resultado de la libertad de expresión, las personas pueden acceder a una gran cantidad de textos y publicar y conocer diferentes puntos de vista. Ciertamente, la multiplicación de recursos genera diversas maneras de leer (Cassany \& Ayala, 2008.).

El advenimiento de las TIC y la internet ha generado cambios sustanciales en cuanto a la cantidad y diversidad de la información, fomentado así lectores interesados en otra clase de textos, con dinámicas diferentes de lectura mediadas por una carga importante de iconos, imágenes y videos. Sería un error, entonces, afirmar que los niños 
y los jóvenes no leen ni escriben. Por el contrario, al estar inmersos en este contexto del siglo XXI y ser nativos digitales sí lo hacen, pero mediante procesos de lectura y escritura diferentes. Ello genera la necesidad de redescubrir estas prácticas de lectura y escritura en soporte digital, más ahora cuando la sociedad del conocimiento acentúa su importancia ya sea porque se promueven mayores formas de comunicación textual o simplemente porque la interacción con la vida cotidiana es mediada en gran parte por una máquina que exige leer y escribir, lo que genera nuevas formas de prácticas sociales (Cassany $\&$ Ayala, 2008).

Surge, entonces, la preocupación por comprender las prácticas de lectura y escritura que emergen de la introducción de tecnologías en escuelas rurales que no contaban con mediaciones tecnológicas y mantenían las tradicionales formas de leer y escribir. ¿Qué pasa con las prácticas de lectura y escritura de los estudiantes cuando se introducen en la escuela la cultura de la información y la tecnología? ¿Qué nuevas formas de lectura y escritura aparecen por la implementación de las TIC en contextos educativos rurales? A partir de estas cuestiones, se formuló la siguiente pregunta que orientó el desarrollo del proyecto de investigación, cuyos resultados se exponen en el presente artículo: ¿qué prácticas de lectura y escritura surgen por la implementación de las TIC en el aula en los estudiantes de escuelas rurales que son dotadas por primera vez con computadores?

Los resultados aquí presentados, se guían por el propósito de caracterizar las prácticas de lectura y escritura que surgen de la implementación de las TIC en el aula y exploran el uso que los estudiantes de escuela rural hacen de ellas en sus procesos de lectura y escritura y cómo en esos contextos se apropian pedagógicamente de estas herramientas. De igual manera, se buscó identificar las habilidades y disposiciones que surgen hacia la lectura y escritura cuando se usan las TIC en un contexto social con un alto índice de analfabetismo y una baja disposición hacia la lectura.

Sumado a esto, en el campo de la educación se ha considerado que el lenguaje tiene una relación directa con el éxito escolar, dado que todo acto educativo es un acto comunicativo y por ende la comprensión está íntimamente ligada a la competencia comunicativa. Lo anterior significa que la lectura y escritura no son exclusivas del área del lenguaje, pues trascienden y se convierten en soportes del aprendizaje, toda vez que leer y escribir permite dialogar, compartir y contrastar saberes; acceder a información escrita y producirla; llegar a acuerdos; entenderse y comprender el mundo.

\section{Método}

El presente artículo expone los resultados alcanzados en el proyecto de investigación llevado a cabo en el año 2013 con veintiuna escuelas rurales de diferentes municipios del departamento de Cundinamarca, que no contaban con herramientas TIC hasta el año 2012, cuando el programa Computadores para Educar del Ministerio de Tecnologías de la Información y las Comunicaciones (Mintic) las dotó con herramientas TIC y formó a los docentes para su apropiación pedagógica. En este contexto, se observaron las prácticas de lectura y escritura de los estudiantes en soporte digital durante una actividad escolar en el aula de clase; cabe aclarar que dicha observación se hizo un año después de implementadas las TIC en el aula.

Por lo anterior, la investigación tuvo un alcance descriptivo. Es decir, buscó presentar las características relevantes y definitorias de la realidad que se va a estudiar en su contexto natural, con el fin de identificar las necesidades de los estudiantes en el acceso a las TIC y su pertinencia en los procesos de lectura y escritura.

Con el fin de caracterizar los usos que hacen los estudiantes de las TIC en sus prácticas de lectura, escritura e interacción, se diseñó una bitácora de observación externa y por ende no participante, puesto que no era factible intervenir, modificar o influir en el normal desarrollo de la clase mediada por las TIC. Por tal razón, el investigador se mantuvo externo a la actividad y solo hasta el final interactuó con los sujetos observados con el fin de ampliar aquella información que no hubiera alcanzado a recabar en la observación.

Se diseñaron unas categorías de análisis que orientaron el diseño de instrumentos y el posterior análisis de resultados. Las variables formuladas se presentan en la Figura 1.

La población objeto de estudio estuvo conformada por los estudiantes de las instituciones educativas rurales que participaron del programa Computadores para Educar de año 2012, en el departamento de Cundinamarca. Esta población fue escogida en virtud de dos características fundamentales que convenían a los objetivos de la investigación: ser escuelas rurales y no haber tenido computadores antes del beneficio otorgado por el programa.

En total 97 sedes cumplieron con estas condiciones, que conformaron un universo del cual se escogió un diseño 
Figura 1

Categorías de análisis

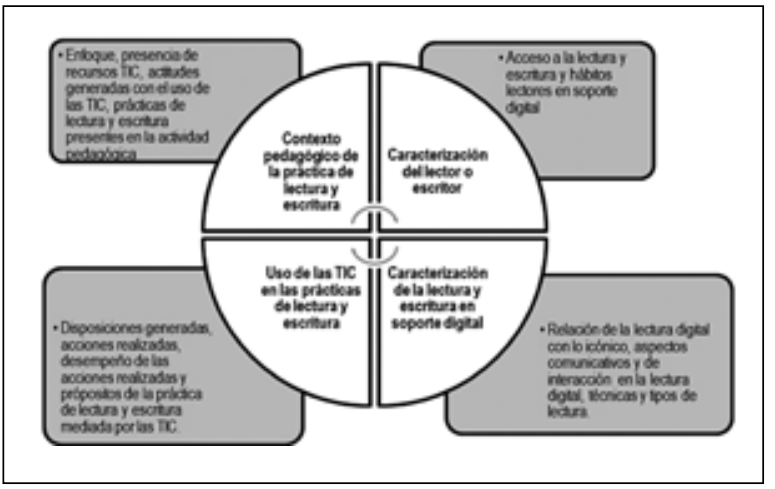

Fuente: Elaboración propia

muestral intencional estratificado. En consideración a que la población es altamente homogénea y por tanto las sedes educativas seleccionadas comparten unidad cultural y étnica, se diseñaron estratos por regiones en Cundinamarca y se seleccionaron de una a tres sedes representantes por región, para completar una muestra de veintiuna sedes educativas.

En la fase de recolección de información se visitaron las veintiuna sedes seleccionadas y en cada una se observaron a todos los estudiantes de la escuela durante una actividad escolar. Los pasos seguidos en la aplicación de la guía de observación fueron los siguientes: a. presentación de la intención de la recolección de información al docente; b. observación de la actividad desde el comienzo hasta su finalización; c. registro en la guía de observación de los datos observados y registro fotográfico; $d$. conversación con el docente y los estudiantes al finalizar la observación para ampliar la información y e. solicitud de autorización para la divulgación de la información recogida.

Para la recolección de los datos se utilizó una guía de observación que permitió estandarizar la aplicación del instrumento. Así, en la guía se delimitaron los aspectos específicos que debían observarse y respondían a cada una de las categorías generales y a los descriptores formulados previamente. Por ejemplo, se observaron concretamente la temática y área del conocimiento en la que se efectuó la práctica de lectura y escritura, sobre la base de que estos procesos no solo se desarrollan en la clase de lenguaje. También se examinaron los tipos de recursos TIC utilizados, la disposición y atención de los estudiantes frente al uso de las TIC, la clase de textos digitales consultados, el tipo de lectura desarrollada, y las actitudes durante el proceso de lectura y escritura en soporte digital, entre otros aspectos.

Para facilitar la recolección de la información y su posterior análisis, se elaboró la guía de observación utilizando escala de medidas como: a. sí y no, para determinar la presencia o ausencia de un aspecto por observar; $b$. ninguno, pocos, muchos y todos, para determinar alguna actitud o acción de los estudiantes; y c. abanico de respuestas para seleccionar los aspectos, emociones y expresiones que reflejaban los estudiantes durante la actividad.

Una vez recolectados los datos, la información se organizó según las categorías diseñadas previamente y que fueron la base para la construcción de la guía de observación. En el análisis, se ponderaron las respuestas y se identificaron tendencias, lo que permitió triangular la información recogida con el marco conceptual. La ponderación hizo posible determinar lo siguiente:

1. Si la práctica de lectura y escritura se efectuó con la finalidad de proporcionar un aprendizaje específico y por ende, las TIC fueron usadas como una herramienta pedagógica y no como un fin de aprendizaje.

2. Si las TIC generaron motivación y disposición para el aprendizaje.

3. El tipo de orientación que los docentes dieron a la práctica de lectura y escritura en soporte digital

4. El nivel de acciones cognitivas desarrolladas con la lectura y escritura mediada por las TIC, que va desde la simple exploración hasta la interpretación y producción.

5. Las capacidades desarrolladas con la lectura en soporte digital, como la autonomía y el pensamiento crítico. En este punto cabe destacar que las preguntas abiertas y la conversación al final con docentes y estudiantes, permitieron ampliar la información recogida, por lo cual fueron categorizadas a la luz de las categorías y descriptores establecidos.

\section{Resultados}

\section{Prácticas de lectura y escritura mediadas por las TIC en las sedes educativas rurales de Cundinamarca}

En su mayoría, los grupos de estudiantes observados fueron pequeńos, con un promedio de 17 estudiantes. De los 21 grupos observados, 13 grupos no superaron los 17 estudiantes, 7 grupos estaban entre 18 y 30 estudiantes y tan solo un grupo grande de 31 estudiantes participó del estudio.

De los 21 grupos, solo dos clases tenían estudiantes de un mismo curso: el grupo de preescolar y un grupo de 
cuarto de primaria. Los 19 grupos restantes se caracterizaron por tener estudiantes de diferentes grados en una misma aula de clase. El área de conocimiento en el que se desarrollaron las 21 actividades pedagógicas observadas, fueron principalmente lenguaje e informática. Así, el 71 $\%$ de los docentes afirmaron estar desarrollando contenidos del lenguaje y el $62 \%$ contenidos de informática (Figura 2).

Es de resaltar que al tener una modalidad escolar de multigrado, se percibió la presencia de un trabajo interdisciplinar entre las áreas básicas, como son matemáticas, lenguaje, ciencias naturales y sociales. En razón al uso de las TIC, los docentes tendieron a responder que además de las áreas básicas trabajaban informática; sólo un $38 \%$ consideró que a pesar de usar las TIC durante la actividad pedagógica, el objetivo era abordar una o varias áreas básicas concretas y ninguno consideró que el uso de las TIC se refería a un trabajo exclusivo de informática. Lo anterior es interesante de observar porque se evidencia cómo en las actividades pedagógicas observadas, el uso de las TIC comienza a ser concebido como una herramienta de enseñanza o mediación para el trabajo de otras áreas básicas, sin reducirlas exclusivamente a la clase de informática.

En total participaron 368 estudiantes en las 21 actividades pedagógicas observadas. De este número, el $12 \%$ tiene computador en su casa y tan solo el $5 \%$ cuenta con acceso a internet. En cuanto al uso del computador en la escuela, los estudiantes afirmaron que en su mayoría leen y escriben en impreso o papel y que en los computadores se tiende a leer más que a escribir (Figura 3)

Es común la ausencia de tecnología en estos contextos, sobre todo si se considera que las escuelas observadas se ubican en la zona rural, donde la brecha digital es aún mayor y no se cuenta con señal masiva de internet. Esto implica que la mayoría de las veces las TIC no forman parte de la cotidianidad de los estudiantes, lo que conduce

Figura 2

Área del conocimiento en la que se desarrolla la actividad pedagógica

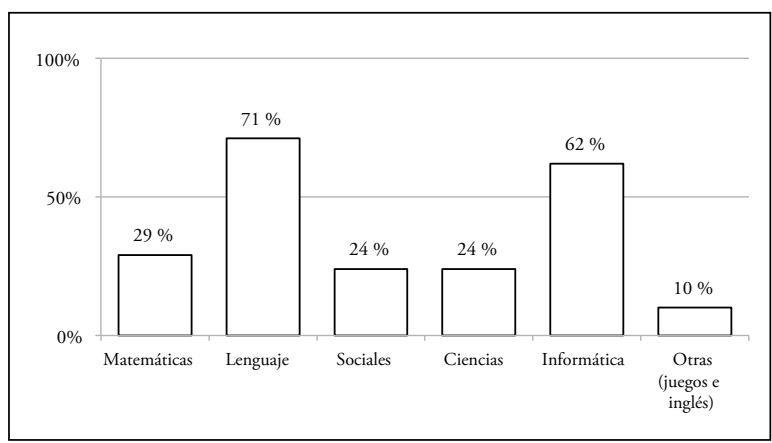

Fuente: Elaboración propia
Figura 3

Soporte en el que los estudiantes leen y escriben en clase

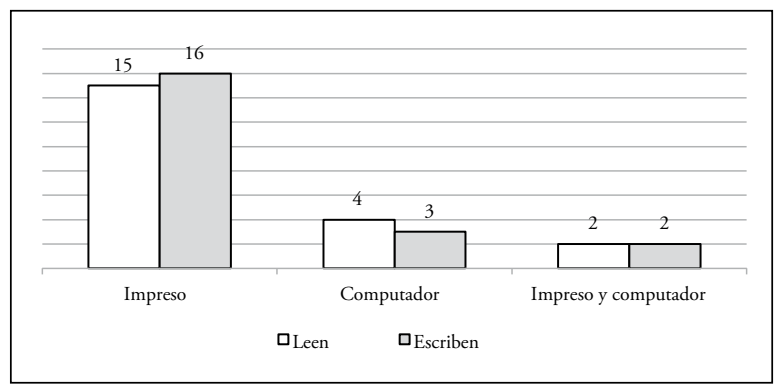

Fuente: Elaboración propia

a que los estudiantes observados no puedan ser clasificados como nativos digitales a pesar de haber nacido en la sociedad del conocimiento. Son inmigrantes digitales y prevalecen en ellos la lectura y la escritura en soporte físico, el trabajo individual y el aislamiento (Cassany \& Ayala, 2008).

Las actividades específicas observadas se resumen en tres grandes categorías: actividades didácticas sobre una temática específica de una de las áreas básicas, en las cuales los procesos de lectura y escritura se dieron para el manejo de la maquina o la resolución de la actividad; actividades específicas de lectura y actividades de producción de textos (Tabla 1).

Tabla 1

Tipo de actividades desarrolladas

\begin{tabular}{ll}
\hline Actividades & -Diligenciamiento de un formulario didáctico \\
didácticas sobre & sobre la temática del cuerpo. \\
un área básica & -CD didácticos en ciencias y geografía; memorizar \\
& departamentos y capitales. \\
& -Juegos con operaciones matemáticas y tablas de \\
& multiplicar. \\
& -Grabar un programa para una emisora. \\
& -Estudio de figuras geométricas por medio de un \\
& programa y colorearlas en Paint. \\
& -Colorear las partes de la planta en Paint y presen- \\
& tarlas en PowerPoint. \\
\hline Procesos de & -Lectura de un texto y resumen sobre él. \\
lectura & -Ver en video beam un cuento sin dialogo e inter- \\
& pretar la secuencia de la historia y los personajes. \\
& -Leer cuentos dentro del proyecto de aula Cuénta- \\
& me un cuento. \\
& -Lectura de fabulas, textos líricos y poesía. \\
& -Pruebas de comprensión de lectura. \\
& -Representación de un texto de PowerPoint por \\
& medio de imágenes. \\
\hline Producción de & -Transcripción de un cuento de un libro a Word \\
texto & utilizando los iconos de negrilla, cursiva, etc. \\
& -Creación de cuentos en Word. \\
& -Producción de autobiografías en Word. \\
& -A partir de un relato, redactar textos para los de \\
& tercero, cuarto y quinto y hacer dibujos para los de \\
primero y segundo.
\end{tabular}

Fuente: Elaboración propia 
De igual manera, estas actividades de enseñanza-aprendizaje utilizando las TIC, fueron orientadas según cuatro objetivos pedagógicos: el mejoramiento de la práctica de lectura y escritura, el aprendizaje de una temática específica de las áreas básicas, el aprendizaje o manejo de las TIC y el desarrollo de habilidades específicas. Los objetivos pedagógicos con base en los cuales los docentes usaban las TIC en su contexto de clase, se observan en el Tabla 2.

Durante las actividades escolares observadas, la TIC que utilizó el $100 \%$ de los grupos fue el computador, un $33 \%$ utilizó el video beam y el $19 \%$ utilizó el celular. En cuanto al uso de herramientas virtuales, en nueve grupos se tuvo internet y en doce no es utilizado debido a la falta de conectividad en la zona. La mayoría, (57\%) acompaña las herramientas virtuales y los recursos TIC con textos físicos, el $43 \%$ con juegos y juguetes y un $33 \%$ no acompañan el uso del computador y video beam con ningún otro recurso.

Durante la actividad pedagógica, se observó que el $90 \%$ de los estudiantes mostró interés por participar y hacer las cosas al comenzar la actividad mediada por las TIC, el $81 \%$ entusiasmo y ánimo y un $62 \%$ tuvo

\section{Tabla 2}

Objetivos de las actividades desarrolladas

\begin{tabular}{|c|c|}
\hline $\begin{array}{l}\text { Objetivos enfoca- } \\
\text { dos a mejorar la } \\
\text { práctica de lectura } \\
\text { y escritura }\end{array}$ & $\begin{array}{l}\text {-Crear un resumen a partir de la lectura que } \\
\text { escucharon. } \\
\text {-Desarrollar habilidades en el manejo de textos. } \\
\text {-Trabajar ortografía. } \\
\text {-Desarrollar expresión oral. } \\
\text {-Desarrollar la escritura. } \\
\text {-Fomentar el aprendizaje de estructuras } \\
\text { narrativas. } \\
\text {-Promover la comprensión de lectura. }\end{array}$ \\
\hline $\begin{array}{l}\text { Objetivos enfoca- } \\
\text { dos al aprendizaje } \\
\text { de una temática } \\
\text { específica }\end{array}$ & $\begin{array}{l}\text {-Aprender sobre el cuerpo, los números, las } \\
\text { figuras geométricas, las operaciones matemáti- } \\
\text { cas, los colores, los valores, etc. } \\
\text {-Ampliar información sobre el tema específico } \\
\text { en los programas. }\end{array}$ \\
\hline $\begin{array}{l}\text { Objetivos enfoca- } \\
\text { dos al aprendizaje } \\
\text { del manejo de la } \\
\text { herramienta TIC }\end{array}$ & $\begin{array}{l}\text {-Trabajar los iconos de Word para texto y } \\
\text { tablas. } \\
\text {-Conocer los iconos de los diferentes progra- } \\
\text { mas. } \\
\text {-Hacer mejoras al documento de color, decora- } \\
\text { ción y tamańo. } \\
\text {-Manejar el programa Paint. } \\
\text {-Exploración de juegos. } \\
\text {-Aprender el uso de herramientas TIC como } \\
\text { PowerPoint. } \\
\text {-Fomentar el manejo de las TIC por parte de } \\
\text { los estudiantes. }\end{array}$ \\
\hline $\begin{array}{l}\text { Objetivos enfoca- } \\
\text { dos al desarrollo } \\
\text { de habilidades } \\
\text { específicas }\end{array}$ & $\begin{array}{l}\text {-Promover la participación de los estudiantes. } \\
\text {-Desarrollar la imaginación y la creatividad. } \\
\text {-Estimular la creación y la composición. }\end{array}$ \\
\hline
\end{tabular}

Fuente: Elaboración propia disposición de atención. Ningún estudiante se opuso a la utilización del computador.

Los grupos que durante la actividad pedagógica usaron herramientas virtuales, muestran una mayor tendencia a consultar juegos (Figura 4) y los participantes que no utilizaron internet, consultaron textos de literatura, escolares y enciclopedias digitales.

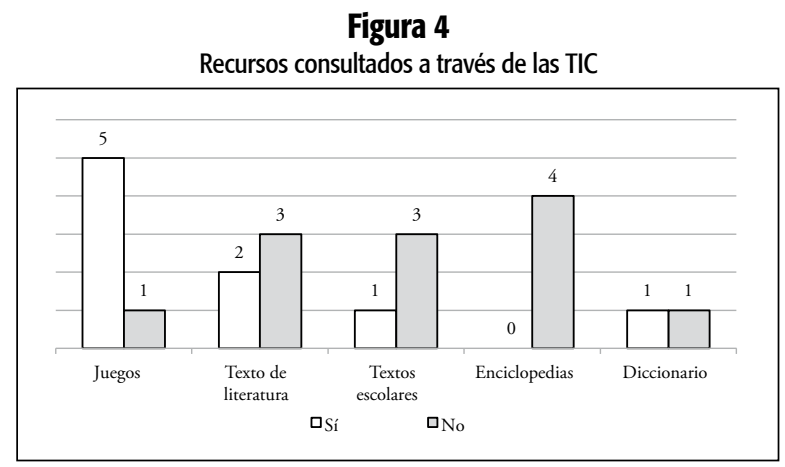

Fuente: Elaboración propia

En cuanto a las actitudes, comportamientos y disposiciones generadas hacia la lectura y la escritura con el uso de las TIC, y a las habilidades de aprendizaje potenciadas con este tipo de herramientas, se observa que el uso de las TIC promovió en dieciocho grupos el interés constante de la mayoría de los estudiantes por las actividades, lo cual se evidenció en la atención permanente en la actividad y sobre todo en su desarrollo completo, con muestras de expresiones de entusiasmo, sorpresa, alegría e interés. Lo anterior fue diferente en dos o cuatro grupos, en los que los estudiantes perdieron la concentración en el transcurso de la actividad o evadieron la lectura y solo prestaron atención al lenguaje icónico o a los videos (Figura 5).

Durante la práctica de lectura y escritura mediada por la TIC, los estudiantes ejecutan con mayor frecuencia acciones de exploración y producción de texto. Se dan

Figura 5

Actitudes de los estudiantes durante la práctica de lectura y escritura en soporte digital

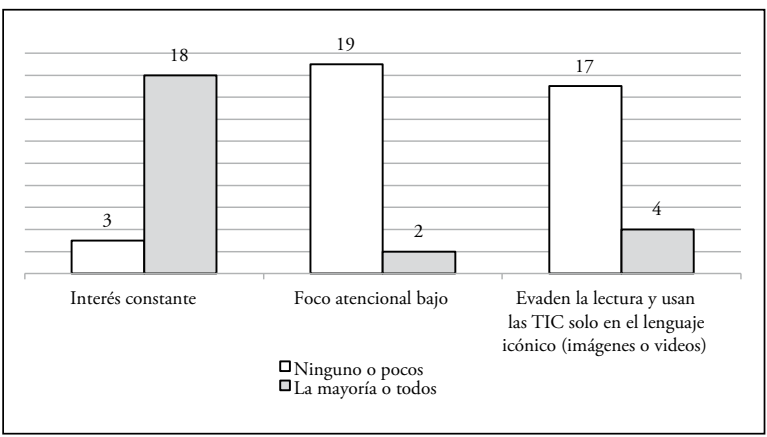

Fuente: Elaboración propia 
pocos procesos de ampliación de información, relación y síntesis (Figura 6).

\section{Figura 6}

Acciones ejecutadas por los estudiantes cuando leen y escriben en soporte digital

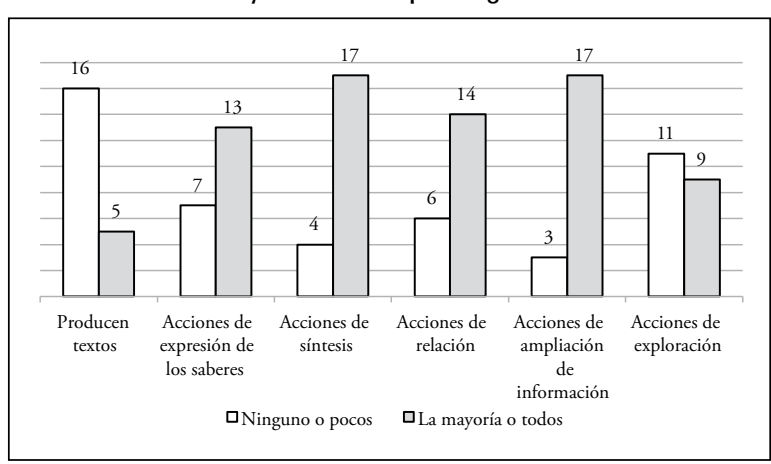

Fuente: Elaboración propia

Lo anterior es debido en gran parte a la ausencia de internet, razón por la cual la actividad siempre estuvo sujeta al recurso de lectura con el que se contaba en el momento. A su vez, esto se liga a los objetivos de la actividad propuestos al comienzo, que orientaron la actividad hacia la enseñanza de la lectura a partir de la codificación y decodificación de la palabra o a la transcripción de textos para la enseñanza de ortografía. Como lo exponen Cassany y Ayala (2008): "el docente debería hacer un cambio de puntos de vista. Es decir, no enseñar la ortografía, sino enseñar cómo la máquina puede usarse para mejorar la ortografía" (p. 67).

La finalidad de la práctica de lectura durante la actividad pedagógica es, principalmente, hacer uso de las TIC, aprender a leer, divertirse y en menor proporción indagar sobre el tema. En cuanto a la práctica de escritura, la finalidad es interactuar con los otros, comunicar las ideas propias sobre el problema, transcribir textos y en menor medida acceder a la información. Esto permitió evidenciar que la ausencia de internet disminuyó una de las fortalezas de lo virtual, como es la ampliación de la información. Aquí no se contó con textos sumativos que permitieran enriquecer el conocimiento y en consecuencia, la lectura en soporte digital y físico no mostró gran diferencia (Figura 7).

La mayoría de los estudiantes que participaron de los grupos de observación, cuando leen en soporte digital por lo general hacen una lectura icónica e intercambian sus impresiones acerca del texto. En once grupos de veintiuno los estudiantes, en su mayoría, solo exploran los textos que están acompañados de videos, imágenes o sonidos y hacen lectura colectiva sobre un mismo texto; lo que aún falta por promover son los procesos de lectura autónoma. Los
Figura 7

Finalidad de la práctica de lectura y escritura en soporte digital

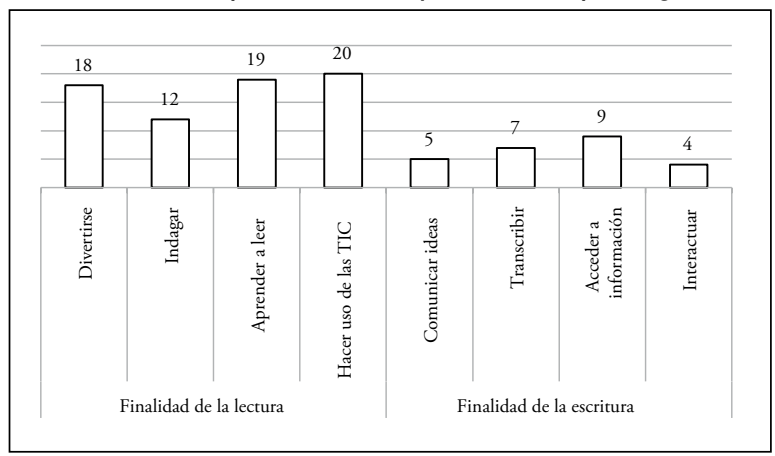

Fuente: Elaboración propia

estudiantes no eligen lo que leen; siempre leen lo que dispone el docente y no consultan otros textos. Esto mostró que los alumnos no llevan a cabo procesos de indagación y búsqueda, como se evidenció anteriormente. A su vez, se hace una lectura dispersa al intercambiar rápidamente de textos y no darles una lectura completa (Figura 8).

\section{Figura 8}

Tipo de lectura y escritura que hacen los estudiantes en soporte digital

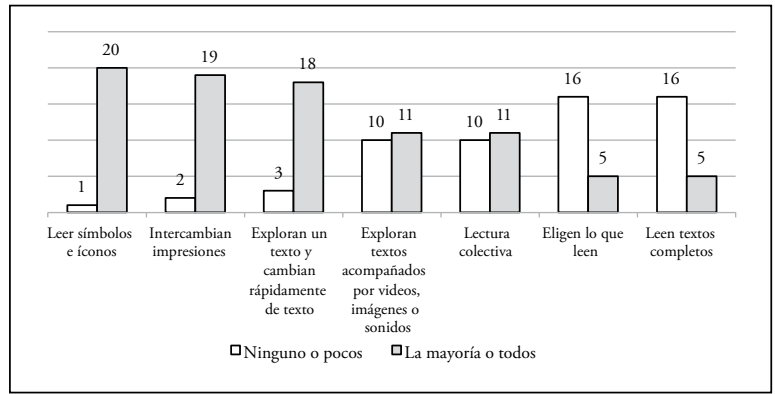

Fuente: Elaboración propia

La técnica de lectura mayormente empleada por los estudiantes durante las prácticas de lectura y escritura observadas, fue la literal; es decir, leyeron de manera secuencial y de principio a fin un mismo texto. Los docentes no indagaban sobre la interpretación que pudieron haberle dado y por lo general se preocuparon por que los estudiantes leyeran de manera fluida y rápida. Esto dejó de lado la lectura intertextual al no profundizar en las temáticas trabajadas o desplazarse de un texto a otro y no elegir aquellos que pudieran ampliar la información. Los estudiantes se limitaron a leer el texto propuesto por el docente y concentrar la actividad en un ejercicio de codificación y decodificación. Por supuesto, tampoco se mostró una lectura inferencial, debido a que los educandos no tuvieron un espacio de participación para expresar su percepción e interpretación del texto y mucho menos se generaron dinámicas de grupo que favorecieran el 
intercambio de ideas entre los mismos estudiantes. En la siguiente figura se resume lo expuesto:

Figura 9

Técnicas de lectura empleadas durante la práctica pedagógica

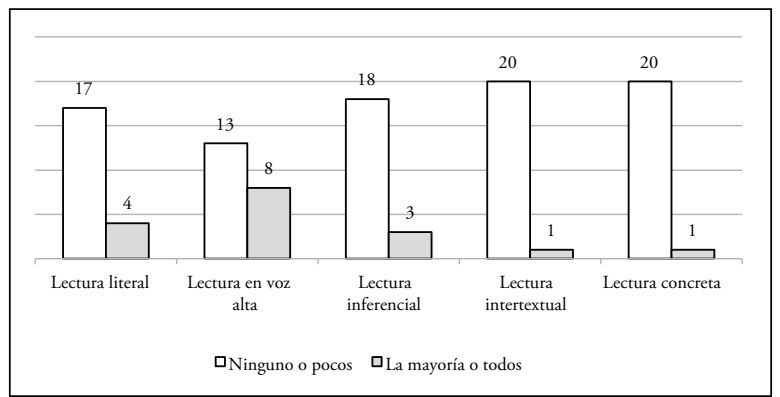

Fuente: Elaboración propia

El papel desempeñado por el docente y sus acciones son fundamentales al momento de promover en los estudiantes aprendizajes importantes mediante el uso de las TIC como herramienta pedagógica. De acuerdo con esto, a continuación se evidencian las acciones que llevó a cabo el docente mientras los estudiantes desarrollaban procesos de lectura y escritura. Entre las principales acciones se destacaron el acompañamiento a los estudiantes y dejarlos explorar solos; otras fue reunir la lectura hecha por cada uno. Se evidenciaron la falta de orientación a los textos leídos, de consulta sobre las interpretaciones de cada uno y el cuestionamiento sobre lo que leen.

\section{Discusión}

\section{Reflexiones sobre las prácticas de lectura y escritura cuando se hace en soporte digital}

Para el caso de este estudio, las escuelas rurales donde se desarrollaron las observaciones se caracterizan por ser unitarias; es decir, un docente orienta el desarrollo curricular de todos los grados mediante la metodología Escuela Nueva o metodología tradicional. En la mayoría de los casos, la población escolar no supera por establecimiento los treinta estudiantes en el ciclo de básica primaria.

La brecha para el acceso al conocimiento global inicia con las dificultades de conectividad, especialmente las relacionadas con el servicio público de energía eléctrica, indispensable para el uso de herramientas informáticas y el cumplimiento del objetivo de mediar los aprendizajes por las tecnologías de la información y la comunicación. La intermitencia en el servicio de fluido eléctrico limita las posibilidades de contar con el uso de las TIC de manera continua y sostenible durante las jornadas escolares. Así, en la mayoría de los grupos observados las prácticas de lectura y escritura en soporte digital no se desarrollan de manera constante y se lee y se escribe principalmente en impreso o papel.

El uso del ordenador y la introducción de las TIC modifican las estructuras educativas, las cuales en el contexto rural, deben adaptarse a las formas de vida que allí se presentan (Itzcovich, 2012). De esta manera, el creciente acceso a la información en cuanto a su rapidez y cantidad y a sus posibilidades de superar la distancia y el tiempo en la comunicación con otros, abre un espacio en la educación decisivo para el futuro. En el contexto rural esto tiene otras connotaciones que obligan a considerar la relación intrínseca entre cultura y tecnología. Como lo expone Castells, (2001), no solo cabe establecer como marco de análisis la cultura escolar en la introducción de las TIC al aula; también es necesario avistar las realidades externas a la escuela en las que el cambio constante y acelerado quebranta los viejos esquemas e impone nuevos moldes, principios y paradigmas (Cabero, 1991).

Así, al observar las veintiuna escuelas rurales, fue claro identificar cómo estos recursos tecnológicos son absorbidos por las prácticas tradicionales de enseñanza sin introducir todos los cambios pronosticados. De esta manera, se percibió que aún falta por parte de los maestros y estudiantes una apropiación más pedagógica de las TIC y no únicamente el manejo utilitario de estas herramientas (Cassany \& Ayala, 2008).

Lo anterior se debe a la idea errónea de que el uso de las TIC en el aula consiste en la simple instalación de un artefacto novedoso, sin considerar que se trata de establecer nuevas funciones sociales mediante la generación de nuevas formas de comunicación, de transmisión del conocimiento y hasta de reproducción del poder (Castells, 2001). No se puede desconocer que los niños disfrutan trabajando en el computador, recurso novedoso para ellos que puede hacer de su experiencia de aprendizaje por medio de las TIC algo altamente significativo. Sin embargo, en los contextos rurales las tecnologías no se encuentran incorporadas a la vida cotidiana ni a los oficios que desarrollan comúnmente. Las condiciones propias del contexto extraescolar exigen la utilización de visiones alternativas para la incorporación de las TIC en el aula rural. De este modo, tan solo el $12 \%$ cuenta con computador en la casa y el $5 \%$ tiene conectividad. Por otro lado, la utilización en el aula de las TIC no es constante por la falta de energía eléctrica y conectividad.

Crook, (1996), propone una perspectiva sociocultural al plantear que el ordenador se convierte en un artefacto cultural que se sitúa y crea para satisfacer necesidades 
específicas. De allí que las actividades escolares lo deban considerar como un medio para obtener aprendizajes dentro de contextos particulares y no un fin en sí mismo o una herramienta utilitarista más que pedagógica. Como expresa Hopenhayn, (2003), los recursos, los libros, los maestros y los programas de estudio, entre otros, muchas veces son producidos fuera del contexto rural y en consecuencia se desarrollan aprendizajes carentes de significado para estudiantes insertos en contextos diversos, razón por la cual es importante adaptar estos recursos y capacidades a las realidades locales. Por tanto, la incorporación de las TIC en el aula no solo se justifica por sus prometedoras bondades; es necesario alinear esta incorporación a las necesidades, los intereses y el contexto propio de la escuela, haciendo de las TIC en el aula una propuesta pedagógica pertinente y relevante, dos condiciones indispensables para la calidad educativa.

Los esfuerzos de las políticas actuales se orientan hacia la integración de las TIC en el aula, para lo cual es necesario que estas herramientas sean dotadas de principios educativos y didácticos y se invisibilicen, de suerte que el aprendizaje siga siendo el objetivo principal. En consecuencia, las TIC se convierten en el vehículo para el desarrollo de contenidos y habilidades (Orjuela, 2012); sin embargo, se observa que aún el uso de la TIC y aprender a manejarlas sigue siendo uno de los objetivos principales en las actividades pedagógicas observadas. A pesar de que se usan para el aprendizaje de otras habilidades y áreas, los maestros afirman que por usarlas se encuentran también en clase de informática y en los objetivos de la actividad siempre hay mención al aprendizaje de la herramienta, lo que muestra que el vehículo no está del todo invisibilizado.

Usar las TIC en el aula implica hacerlo para una diversidad de fines, como lograr el aprendizaje de un concepto, un proceso o una disciplina curricular. Esto significa encontrar las posibilidades didácticas de las TIC en relación con los objetivos y los fines educativos (Sánchez, 2009). En el caso de los grupos observados, aunque las actividades pedagógicas tenían diferentes objetivos -como el mejoramiento de la práctica de lectura y la escritura o el aprendizaje de una temática específica de las áreas básicas-, los docentes no invisibilizan las herramientas tecnológicas y lo que hacen es crear un objetivo específico dentro de la actividad pedagógica como es el aprendizaje o el manejo de las TIC. Lo anterior significa que los docentes están trabajando de manera simultánea los propósitos de aprendizaje con los propósitos de manejo de las TIC, pero aún les falta llegar a una integración real de las áreas básicas con el uso de estas herramientas como facilitadoras del aprendizaje.
Las formas de leer van íntimamente ligadas a la historia del libro o a la tecnología con la que se presentan los textos. Si nos remontamos a los siglos anteriores, se ve cómo la transformación de la presentación del libro impacta en la "producción del lector" (Andrade, 2007). Entre el siglo segundo y cuarto, con el surgimiento de la imprenta en la época moderna, el público lector se amplió, hubo mayor familiarización con los libros gracias a la multiplicación de los ejemplares y estos se tradujeron a otras lenguas. Con la aparición del texto electrónico se dan cambios e incluso rupturas; el texto electrónico es móvil, maleable, abierto y el lector puede intervenir en su contenido mismo (Snyder, 2004).

Por lo tanto, el soporte digital ofrece un acceso mayor a la información, pero en esa misma proporción debe aumentar la capacidad de control y el análisis de la información, puesto que los contenidos no siempre están sujetos al dominio riguroso y a la intervención de una editorial. La cantidad de información exige una mayor capacidad de comprensión para dotarla de sentido y discriminar entre lo verdadero y lo falso; entre lo válido y lo inválido.

En la observación de las prácticas de lectura y escritura, se evidenció que a pesar de usar mediaciones TIC, se siguen manteniendo los procesos de lectura y escritura tradicionales basados en la memoria y se deja de lado la comprensión para enfocarse en la memorización del alfabeto. Por lo tanto, en la enseńanza de la lectura y la escritura persiste la concepción codificadora y decodificadora de símbolos, la identificación de ideas principales y la repetición de textos, a diferencia de lo que propone el Ministerio de Educación en el sentido de que el aprendizaje de la lectura y la escritura es

[...] el instrumento básico de la interacción humana, y todos los aprendizajes se basan en esa interacción. Es un universo de significados que permite interpretar el mundo y transformarlo, construir nuevas realidades, establecer acuerdos para poder convivir con los congéneres y expresar ideas y sentimientos (MEN, 1998).

Otro factor característico de la lectura en soporte digital es la promoción de la lectura hipertextual que rompe la estructura tradicional del escrito. Esta lectura no se hace de manera lineal -es decir, empezando por la primera página hasta llegar al último párrafo- sino que el hipertexto permite ampliar la información sobre algún concepto o tema específico con solo dar clic a la palabra resaltada. Un enlace lleva a otro escrito; una imagen ilustra el contenido; un sonido amplía el concepto; de esta manera el tema se hace más didáctico y pedagógico (Alcalá-Caldera $\&$ Rasero-Machacón, 2004). Sin embargo, esto tampoco 
fue observado en los grupos dado que en su mayoría los estudiantes seguían desarrollando -por proposición del docente- una lectura literal, aunque estuvieran leyendo en soporte digital.

Así, la organización arbórea de los textos producto del desarrollo tecnológico en contextos como el observado no ha logrado diversificar las prácticas de lectura, lo cual ha hecho perder la capacidad de conectar un texto con otro y por ende se disminuye el potencial de ampliar la información o contrastar con la multiplicidad de sentidos y enfoques. La lectura y la escritura en soporte digital, pone sobre la mesa la diversidad de recursos dispuestos en los procesos de comunicación en los que se generan diversas formas de representación y construcción de sentido. Por ello, la inclusión adecuada de la tecnología en el aula exige el desarrollo de un conocimiento múltiple, articulado y contextualizado (Cano, 2010).

Una de las principales diferencias entre un lector de libros impresos y uno de textos digitales radica en el contexto. En la lectura en soporte físico la construcción del sentido de cada texto depende de su relación con los referentes que lo anteceden o preceden, estos referentes fueron reunidos un mismo objeto impreso con una intención editorial específica. En cambio, la lectura en soporte digital se presenta a partir de una organización enciclopédica del saber que propone al lector textos sin otro contexto que el de su pertenencia a una misma temática (Cassany \& Ayala, 2008).

Estas diferencias son importantes no porque la lectura electrónica esté amenazando de muerte a la lectura o al libro, sino porque pone de presente los nuevos retos que surgen al momento de identificar lo que implican las nuevas técnicas de inscripción difusión y apropiación de los textos. La revolución del texto electrónico es, asimismo, una revolución de la lectura; por lo tanto, la revolución actual no modifica solo las técnicas de reproducción del texto, sino también la transformación en los modos de organización y estructuración de lo escrito (Chartier, citado en Andrade, 2007).

Actualmente, el aprendizaje de la lectura y la generación de hábitos de lectura se encuentran ante un nuevo paradigma de enseñanza marcado por las TIC, herramientas que hacen posible un fácil acceso a la información y aportan procesos rápidos y flexibles de datos, canales de comunicación inmediata, capacidad de almacenamiento, automatización de trabajos, interactividad y digitalización de la información (Alcalá-Caldera \& Rasero-Machacón, 2004).
Las TIC generan aspectos positivos para el desarrollo de prácticas de lectura y escritura, en especial en contextos rurales multigrados e interdisciplinares. Algunos de estos aspectos son la manera de almacenar, que permite a los estudiantes conectar sus ideas a lo largo de un tiempo extenso; la retroalimentación o colaboración de pares; el desarrollo de diferentes lenguajes gracias a la multimedia, y sobre todo la participación activa que extiende las posibilidades de aprendizaje más allá de las que se ofrecen en clase. Igualmente, la convergencia de medios, la hipertextualidad y el alto grado de interactividad que generan las TIC, crean espacios de trabajo autogestionados y colaborativos que repercuten positivamente en la comprensión y producción de textos (Álvarez, 2012). En cuanto a la práctica de escritura, cuando se escribe en procesadores es más fácil identificar errores ortográficos y encontrar sinónimos y antónimos que enriquecen el vocabulario y tomar conciencia de los errores, entre otros aspectos (Ortiz, 2004).

En el caso de los estudiantes que formaron parte de este estudio, fue evidente la motivación que se suscita en ellos por participar y trabajar en clase gracias al uso de las TIC. Estas promueven el trabajo colaborativo en un aula multigrado y como herramienta pedagógica favorecen el desarrollo de una metodología apropiada -específicamente en el fomento del trabajo colaborativo- que se da gracias a la facilidad de los procesos de comunicación e interacción entre los estudiantes con los diferentes contenidos. Además, se facilita el aprendizaje autónomo y la ejecución de las tareas según el ritmo de cada estudiante.

Sin embargo, cabe resaltar una dificultad importante que se presenta con la implementación de las TIC en el aula, específicamente en las prácticas de lectura y escritura. Al no contar con conectividad, al momento que los estudiantes leen y escriben en soporte digital se reproducen las limitaciones propias de la lectura en soporte físico, dado que se pierden el proceso autónomo, la lectura hipertextual, la capacidad de exploración y búsqueda, y la ampliación de información, entre otros. Esto hace que se malogre la transversalidad que se genera con el uso de herramientas tecnológicas y se pierda la oportunidad de acceder a contenidos digitales que podrían fortalecer el currículo.

Por su parte, durante la práctica de lectura y escritura los estudiantes tomaron la iniciativa en el proceso. En la lectura en voz alta o durante el desarrollo de una actividad, se evidenciaba la alta participación de los estudiantes. Particularmente, los estudiantes seleccionaban juegos o actividades didácticas acompañadas de imágenes y videos, 
lo que llevó a concluir que en soporte digital se da una mayor tendencia a la lectura icónica.

En cuanto a los docentes, la observación muestra que aún no tienen apropiadas las TIC como herramienta pedagógica, lo que genera prácticas impropias como dejar que los estudiantes exploren solos la información sin una lectura inferencial y sin el acompañamiento del docente durante el desarrollo de la práctica de lectura y escritura. Esto lleva a un desaprovechamiento de las TIC como herramientas pedagógicas.

A manera de conclusión, cabe afirmar que gracias a la disposición y motivación de los estudiantes para hacer las actividades en el aula, se afianzaron y aclararon de manera didáctica algunos conceptos académicos. Sin embargo, es importante resaltar que la falta de acompañamiento del docente hace que en ocasiones las TIC se conviertan en un foco de distracción y por ende se pierde el propósito de la actividad. En general, los estudiantes manifestaron que las actividades pedagógicas didácticas y digitales les permiten aprender de manera fácil y agradable.

\section{Referencias}

Alcalá-Caldera, J., \& Rasero-Machacón, J. (2004). El papel de las TIC en la animación a la lectura. Revista Lationamericana de Tecnología Educativa, 3(1), 395-416.

Álvarez, G. (2012). Entornos virtuales de aprendizaje y didáctica de la lengua: dos experiencias con integración de TIC para mejorar las habilidades de lectura y escritura de estudiantes preuniversitarios. Educación, Comunicación y Tecnología, 6(12), 1-22.

Andrade, L. (2007). Leer: laboriosa construcción de una práctica y los desafíos de la "ciberlectura". Revista Iberoamericana de Educación, 2(43), 1-17.

Cabero, J. (1991). Actitudes hacia los ordenadores y la Informática. Revista Electrónica de Tecnología Educativa. Recuperado de http://goo.gl/Bwlkm7
Cano, F. (2010). Leer y escribir con las nuevas tecnologías. Rosario: Homo Sapiens.

Cassany, D., \& Ayala, G. (2008). Nativos e inmigrantes digitales en la escuela. Revista Participación Educativa, 9, 53-71.

Castells, M. (2001). La galaxia internet. Madrid: Plaza y Janés.

Crook, Ch. (1996). Ordenadores y aprendizaje colaborativo. Madrid: Ediciones Morata.

Hopenhayn, M. (2003). Educación, comunicación y cultura en la sociedad de la información: una perspectiva latinoamericana. Revista de la Cepal, 81, 175-193.

Itzcovich, G. (2012). Configuraciones espaciales de escenarios urbanos y rurales. Desafios pendientes en los procesos de inclusión educativa. Recuperado de http://goo.gl/kqhStf

Orjuela, D. (2012). Acercamiento a la integración curricular de las TIC. Praxis y Saber, 1(2), 111-136.

Ortiz, O. (2004). Lectura y escritura en la era digital. Desafíos que la introducción de las TIC impone a la tarea de estimular el desarrollo del lenguaje en niños jóvenes. Revista electrónica de Tecnología Educativa. Recuperado de http:// goo.gl/M3kZ9

República de Colombia. Ministerio de Educación Nacional. (1998). Lineamientos curriculares. Lengua Castellana. Recuperado de http://goo.gl/yVSYPT

Sánchez, J. (Noviembre, 2009) Integración curricular de las TICs: Conceptos e Ideas. Trabajo presentado en el congreso Iberoamericano de informática educativa, España.

Sigalés, C., Momino, J. M., Meneses, J., \& Badia, A. (2008). La integración de internet en la educación escolar española: situación actual y perspectiva de futuro. Recuperado de http:// goo.gl/I6UX2P

Snyder, I. (2004). Alfabetismos digitales. Comunicación, innovación y educación en la era electrónica. Málaga: Ediciones Aljibe. 